\title{
AN INDEX THEOREM FOR MODULES ON A HYPERSURFACE SINGULARITY
}

\author{
RAGNAR-OLAF BUCHWEITZ AND DUCO VAN STRATEN
}

To the memory of V. I. Arnol'd

\begin{abstract}
A topological interpretation of Hochster's Theta pairing of two modules on a hypersurface ring is given in terms of linking numbers. This generalizes results of M. Hochster and proves a conjecture of J. Steenbrink. As a corollary we get that the Theta pairing vanishes for isolated hypersurface singularities in an odd number of variables, as was conjectured by H. Dao.
\end{abstract}

\section{INTRODUCTION}

The interplay between topology and algebra is a central theme in singularity theory. The formula expressing the Milnor number of an isolated hypersurface singularity in terms of the length of its local algebra and the Eisenbud-Levine theorem expressing the topological degree as the index of a quadratic form on the local algebra are cases in point. In this paper we prove a theorem in the same spirit: we will show that a purely algebraic quantity, Hochster's Theta pairing associated to two modules on the ring of an isolated hypersurface singularity, can be expressed as a linking number of two associated cycles. In this respect it is reminiscent of the classical interpretation of the intersection multiplicity in terms of linking, due to Lefschetz. The main ideas of this theorem were developed about 25 years ago, during a visit of the first author to Leiden, where he lectured on the triangulated structure of the stable category of maximal Cohen-Macaulay modules, $[\mathrm{B}$. On that occasion, J. Steenbrink came up with a surprising conjecture relating Hochster's Theta pairing to the variation mapping in the cohomology of the Milnor fibre.

The past years have seen renewed interest in maximal Cohen-Macaulay modules and matrix factorisations after the relation with $D$-branes and mirror symmetry in Landau-Ginsburg models was suggested by M. Kontsevich and established by D. Orlov, $\mathrm{O}$. The physics literature on the subject is rather enormous by now. The Kapustin-Li formula $\mathrm{KL}$ implies still another algebraic expression for the Theta pairing, however the main result of this paper still seems to be new.

The structure of the paper is as follows. In the first section we review basic notation and definitions that we need. In the second section we formulate our main theorem. In the third section we use higher algebraic $K$-theory to define, for a module $M$ on a hypersurface ring, a class $\{M\}$ in $K^{1}$ and reformulate Hochster's Theta pairing in $K$-theoretic terms. In the fourth section we map everything to topological $K$-theory and give a different description for $\{M\}_{\text {top }}$. The vanishing of $\theta(M, N)$ for an odd

Date: November 8, 2018. 
number of variables follows from the fact that in that case the Milnor fibre has only even cohomology. This confirms a conjecture formulated in $\mathrm{D}$. that was proven in the case of quasi-homogeneous singularities and graded modules in [MPSW], and recently established as well in our context by Polishchuk and Vaintrob [PV] from the (DG-)categorical point of view. In the fifth section we use the Chern-character to map topological $K$-theory to cohomology, which then leads to a proof of our main result. In a way our result represents a (rather hard won) triumph of topology over algebra that Arnol'd might have appreciated.

\section{Preliminaries}

Topology of singularities. We consider an isolated hypersurface singularity

$$
f \in P:=\mathbb{C}\left\{x_{0}, x_{1}, \ldots, x_{n}\right\}, \quad f(0)=0
$$

and we will choose a good representative $\mathrm{AGV},[\mathrm{LO}$

$$
f: X \longrightarrow D
$$

for the defining function in the usual way: first we take a sufficiently small ball $B(0, \epsilon) \subset \mathbb{C}^{n+1}$ such that the spheres $\partial B\left(0, \epsilon^{\prime}\right)$, for $0<\epsilon^{\prime} \leq \epsilon$, are transverse to $f^{-1}(0)$, then put $X:=B(0, \epsilon) \cap f^{-1}(D)$, where $D:=D_{\eta}$ is the $\eta$-disc in $\mathbb{C}$. Here $\eta$ is taken so small that all fibres $X_{t}:=f^{-1}(t), t \in D$ are transverse to the Milnor sphere $S:=\partial B(0, \epsilon)$. The fibre $X_{0}$ has an isolated singularity in 0 and the restriction $f^{*}: X^{*}:=X \backslash X_{0} \longrightarrow D^{*}:=D \backslash\{0\}$ of $f$ is a locally trivial fibre bundle, the Milnor fibration. The Milnor-fibres $X_{t}:=f^{*-1}(t)$ have the homotopy type of a wedge of $n$-spheres, where the number $\mu(f)$ of these can be computed algebraically as

$$
\mu(f)=\operatorname{dim}_{\mathbb{C}}\left(P / J_{f}\right),
$$

the quotient of the power series ring modulo the jacobian ideal of $f$,

$$
J_{f}:=\left(\frac{\partial f}{\partial x_{0}}, \frac{\partial f}{\partial x_{1}}, \ldots, \frac{\partial f}{\partial x_{n}}\right) \subseteq P .
$$

The singular fibre $X_{0}$ is homeomorphic to the cone over the manifold $L:=S \cap$ $X_{0}=\partial X_{0}$, the link of the singularity, that is homotopy equivalent to the complex manifold $U:=X_{0} \backslash\{0\}$. By the triviality of the Milnor fibration near the boundary, one can identify $L$ also with the boundary $\partial X_{t}$, for any $t \in D^{*}$. From the homology sequence

$$
0 \longrightarrow H_{n}(L) \longrightarrow H_{n}(F) \longrightarrow H_{n}(F, \partial F) \longrightarrow H_{n-1}(L) \longrightarrow 0
$$

of the pair $\left(X_{t}, \partial X_{t}\right)$, where we have written $F=X_{t}$ for the typical Milnor fibre, we see that $L$ has non-trivial homology groups only in degree $n-1$ and $n$ and that these are put in duality by the intersection product. For more details we refer to AGV] and Lo.

Modules over Hypersurface rings. We will consider (finitely generated) modules $M$ over the hypersurface ring $R:=P /(f)$, a local ring of dimension $n$. Typical modules arise by considering subvarieties $Z$ lying inside the singular fibre $X_{0}$. Al-

gebraically, we consider the ideal $I \subset P$ of $Z$, and as $Z$ is supposed to be contained in $X_{0}$, one has $f \in I$. The subvariety $Z$ now determines a module

$$
\mathcal{O}_{Z}:=P / I \text {. }
$$


It is a basic fact, discovered by Eisenbud [E], that $R$-modules have a minimal resolution that is eventually 2-periodic. In fact, a choice of generators for $M$ defines a surjection of a free $R$-module $F$ onto $M$, with the syzygy module $\operatorname{syz}(M)$ as kernel:

$$
0 \longrightarrow \operatorname{syz}(M) \longrightarrow F \longrightarrow M \longrightarrow 0 \text {. }
$$

It follows from the depth-lemma that $\operatorname{depth}_{R}(\operatorname{syz}(M))>\operatorname{depth}_{R}(M)$ as long as $\operatorname{depth}_{R}(M)<n$. Repeating the procedure with $\operatorname{syz}(M)$, we see that after $n$ steps we have an exact sequence of the following form

$$
0 \longrightarrow M^{\prime} \longrightarrow F_{n-1} \longrightarrow F_{n-2} \longrightarrow \cdots F_{0} \longrightarrow M \longrightarrow 0,
$$

where the $F_{i}$ are free $R$-modules of finite rank and $\operatorname{depth}_{R}\left(M^{\prime}\right)=n$. If $M^{\prime}=0$, then $M$ has a free resolution of finite length, if $M^{\prime} \neq 0$, then $M^{\prime}$ is a maximal Cohen-Macaulay module, that is, $\operatorname{depth}_{R}\left(M^{\prime}\right)=n$. So, "up to free modules", any $R$-module $M$ can be "replaced" by a maximal Cohen-Macaulay module. For a systematic account of such Cohen-Macaulay approximations we refer to $[\mathrm{B}], \mathrm{AB}$. If $M$ is a maximal Cohen-Macauly $R$-module that is minimally generated by $p$ elements, its resolution as $P$-module has the form

$$
0 \longrightarrow P^{p} \stackrel{A}{\longrightarrow} P^{p} \longrightarrow M \longrightarrow 0,
$$

where $A$ is some $p \times p$-matrix, with $\operatorname{det}(A)=f^{q}$. Expressing the fact that multiplication by $f$ acts as 0 on $M$ produces a matrix $B \in \operatorname{Mat}(p \times p, P)$, as in the diagram

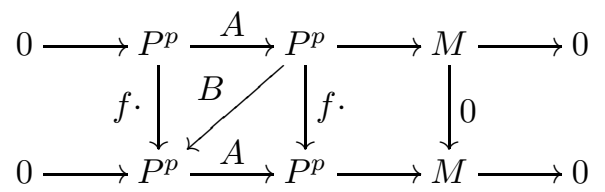

such that

$$
A \cdot B=B \cdot A=f \cdot I,
$$

where $I$ is the identity matrix. In other words, we find a matrix factorisation $(A, B)$ of $f$, determined uniquely, up to base change in the free modules $P^{p}$, by $M$. This matrix factorisation not only determines $M$, as $M=\operatorname{Coker}(A)$, but also determines a resolution of $M$ as $R$-module

$$
\cdots \longrightarrow R^{p} \stackrel{A}{\longrightarrow} R^{p} \stackrel{B}{\longrightarrow} R^{p} \stackrel{A}{\longrightarrow} R^{p} \longrightarrow M \longrightarrow 0
$$

that is plainly 2-periodic. So the minimal resolution of an $R$-module looks in general as follows:

$$
\cdots \longrightarrow G \stackrel{A}{\longrightarrow} F \stackrel{B}{\longrightarrow} G \stackrel{A}{\longrightarrow} F \longrightarrow F_{n-1} \longrightarrow \cdots \longrightarrow F_{0} \longrightarrow M \longrightarrow 0,
$$

where $F=G$ are free $R$-modules. As a consequence, for modules $M$ and $N$ over hypersurface rings, all homological algebra invariants like $\operatorname{Tor}_{k}^{R}(M, N)$ and $\operatorname{Ext}_{R}^{k}(M, N)$ are eventually 2-periodic. It will be convenient also to consider the so-called complete resolution $C^{\bullet}(M)$ of $M$, that is the bi-infinite 2-periodic complex

$$
C^{\bullet}(M): \quad \cdots \stackrel{B}{\longrightarrow} G \stackrel{A}{\longrightarrow} F \stackrel{B}{\longrightarrow} G \stackrel{A}{\longrightarrow} F \stackrel{B}{\longrightarrow} \cdots,
$$

\footnotetext{
${ }^{1}$ at least when $f$ is irreducible. In general, $\operatorname{det} A$ will divide $f^{q}$, with the exponent of an irreducible factor of $f$ in $\operatorname{det} A$ equal to the rank of $M$ on the corresponding component; see [E].
} 
where by convention we always will put $F$ in even and $G$ in odd spots, although always $F=G$. For details we refer to $[\mathrm{E}, \mathrm{Y}], \mathrm{B}$.

Hochster's Theta pairing. The following quantity was considered by Hochster in $[\mathrm{H}]$. In $[\mathrm{MPSW}]$ it is called Hochster's Theta invariant.

Definition: The Theta pairing of modules $M$ and $N$ over the hypersurface ring $R=P /(f)$ is

$$
\theta(M, N):=\operatorname{length}\left(\operatorname{Tor}_{\text {even }}^{R}(M, N)\right)-\operatorname{length}\left(\operatorname{Tor}_{\text {odd }}^{R}(M, N)\right),
$$

where

$$
\operatorname{Tor}_{\text {even }}^{R}(M, N):=\operatorname{Tor}_{2 k}^{R}(M, N), \quad \operatorname{Tor}_{\text {odd }}^{R}(M, N):=\operatorname{Tor}_{2 k+1}^{R}(M, N), \quad k \gg 0 .
$$

(It is enough to take $2 k>n$, as resolutions in those degrees are $2-$ periodic.) This definition makes sense, as soon as the lengths appearing are finite. This certainly happens if $R$ has an isolated singular point, but more generally as soon as one of the two modules is locally free away from the singular point.

Examples 1.1. Hochster's Theta pairing is easy to compute. Let us take the simplest possible singularities, of type $A_{1}$ in two or three variables, and the simplest modules on them:

(i) Take $f=x y, M=\mathbb{C}\{x, y\} /(x), N=\mathbb{C}\{x, y\} /(y), K=\mathbb{C}\{x, y\} /(x, y)$. The resolution of $M$ as $R=\mathbb{C}\{x, y\} /(x y)$-module is

$$
\cdots \longrightarrow R \stackrel{x}{\longrightarrow} R \stackrel{y}{\longrightarrow} R \stackrel{x}{\longrightarrow} R \longrightarrow M \longrightarrow 0,
$$

thus, the "matrix factorization" is simply the factorization $x \cdot y$ of $f$ itself. The $\operatorname{Tor}_{k}^{R}(M, M)$ are computed by tensoring the above with $M$, that is, "going mod $x$ ", and we obtain

$$
\cdots \longrightarrow \mathbb{C}\{y\} \stackrel{0}{\longrightarrow} \mathbb{C}\{y\} \stackrel{y}{\longrightarrow} \mathbb{C}\{y\} \stackrel{0}{\longrightarrow} \mathbb{C}\{y\}
$$

Hence we find

$$
\operatorname{length}\left(\operatorname{Tor}_{\text {even }}^{R}(M, M)\right)=0, \quad \text { length }\left(\operatorname{Tor}_{\text {odd }}^{R}(M, M)\right)=1,
$$

so that $\theta(M, M)=-1$. To get $\theta(M, N)$, we have to "go mod $y$ " instead and obtain

$$
\cdots \longrightarrow \mathbb{C}\{x\} \stackrel{x}{\longrightarrow} \mathbb{C}\{x\} \stackrel{0}{\longrightarrow} \mathbb{C}\{x\} \stackrel{x}{\longrightarrow} \mathbb{C}\{x\}
$$

so that now

$$
\text { length }\left(\operatorname{Tor}_{\text {even }}^{R}(M, N)\right)=1, \quad \operatorname{length}\left(\operatorname{Tor}_{\text {odd }}^{R}(M, N)\right)=0,
$$

hence $\theta(M, N)=1$. Similarly, by going " $\bmod (x, y) "$, one finds each of $\operatorname{Tor}_{\text {even } / \text { odd }}^{R}(M, K)$ to be one-dimensional, whence $\theta(M, K)=0$.

(ii) Take $f=x y-z^{2}, M=\mathbb{C}\{x, y, z\} /(x, z)$. (Note that $M=\mathcal{O}_{L}$, where $L$ is the line $x=z=0$ on the quadric cone $f=0$.) A matrix factorisation $(A, B)$ associated to $M$ is given by

$$
A=\left(\begin{array}{cc}
y & -z \\
-z & x
\end{array}\right), B=\left(\begin{array}{cc}
x & z \\
z & y
\end{array}\right),
$$

and $\operatorname{Tor}_{k}^{R}(M, M)$ is the homology of the complex

$$
\cdots \longrightarrow \mathbb{C}\{y\}^{2} \stackrel{\alpha}{\longrightarrow} \mathbb{C}\{y\}^{2} \stackrel{\beta}{\longrightarrow} \mathbb{C}\{y\}^{2} \stackrel{\alpha}{\longrightarrow} \mathbb{C}\{y\} \longrightarrow \cdots,
$$


where

$$
\alpha=\left(\begin{array}{ll}
y & 0 \\
0 & 0
\end{array}\right), \beta=\left(\begin{array}{ll}
0 & 0 \\
0 & y
\end{array}\right)
$$

So we find

$$
\operatorname{length}\left(\operatorname{Tor}_{\text {even }}^{R}(M, M)\right)=1, \quad \operatorname{length}\left(\operatorname{Tor}_{\text {odd }}^{R}(M, M)\right)=1,
$$

hence $\theta(M, M)=0$.

We list some properties that follow immediately from the definition.

\section{Properties of Hochster's Theta pairing:}

(i) $\theta(M, N)=0$ if $M$ or $N$ is a free $R$-module.

(ii) $\theta(M, N)=\theta(N, M)$.

(iii) $\theta(M, N)$ is additive over short exact sequences: if

$$
0 \longrightarrow M^{\prime} \longrightarrow M \longrightarrow M^{\prime \prime} \longrightarrow 0
$$

is a short exact sequence of $R$-modules, then

$$
\theta(M, N)=\theta\left(M^{\prime}, N\right)+\theta\left(M^{\prime \prime}, N\right) .
$$

(iv) $\theta(M, N)=-\theta(\operatorname{syz}(M), N)$, where $\operatorname{syz}(M)$ is the first syzygy of $M$.

(v) $\theta(M, N)=0$ if either $M$ or $N$ is of finite projective dimension.

(vi) $\theta(M, N)=0$ if either $M$ or $N$ is artinian.

All properties, except maybe the last, are obvious. For (vi), note first that with $m=\left(x_{0}, \ldots, x_{n}\right) R$ the maximal ideal of the ring,

$$
\theta(M, R / m)=\operatorname{rank}(F)-\operatorname{rank}(G)=0,
$$

as tensoring a minimal resolution of $M$ with $R / m$ kills all differentials, and so length $\left(\operatorname{Tor}_{\text {even }}^{R}(M, R / m)\right)=\operatorname{rank}(F)$ and $\operatorname{length}\left(\operatorname{Tor}_{\text {odd }}^{R}(M, R / m)\right)=\operatorname{rank}(G)$. For the general case, we can use induction on the length of $N$, as any artinian $N$ sits in an exact sequence

$$
0 \longrightarrow R / m \longrightarrow N \longrightarrow N^{\prime} \longrightarrow 0
$$

We see that $\theta$ descends to a symmetric pairing on the $K$-group of the category $\bmod (R)$, divided out by the classes of the free and artinian modules.

Remark 1.2. In $[\mathrm{B}]$ the quantity

$$
h(M, N):=\operatorname{length}\left(\operatorname{Ext}_{R}^{\text {even }}(M, N)\right)-\operatorname{length}\left(\operatorname{Ext}_{R}^{\text {odd }}(M, N)\right)
$$

was studied and called the Herbrand difference, as the additive analogue of the Herbrand quotient that arises in group cohomology for representations of cyclic groups, modules over the (integral) "hypersurface" $x^{n}=1$. While it is more convenient for us here to work with Tor, one has the relation

$$
h(M, N)=\theta\left(M^{*}, N\right),
$$

where $M^{*}=\operatorname{Hom}_{R}(M, R)$ is the dual module and $M$ is maximal Cohen-Macaulay, whence the two notions are indeed equivalent, taking into account that $M \cong M^{* *}$. 
2. The meaning of $\theta(M, N)$

An interesting case arises when $M=\mathcal{O}_{Y}=R / I ; N=\mathcal{O}_{Z}=R / J$, where $Y, Z \subset X_{0}$ are subspaces of $X_{0}$, defined by ideals $I$ and $J$ respectively. By additivity over short exact sequences and using the fact that every module admits a finite filtration with sub-quotients of the form $R / I$, knowing all $\theta\left(\mathcal{O}_{Y}, \mathcal{O}_{Z}\right)$ determines $\theta(M, N)$ for all modules $M$ and $N$.

In analogy with Serre's Tor-formula, $\theta\left(\mathcal{O}_{Y}, \mathcal{O}_{Z}\right)$ should have something to do with intersections taking place inside $X$, the domain of $f$. The aim of this paper is to clarify this relation.

Theorem $2.1([\mathrm{H}])$. In the above situation,

$$
\theta\left(\mathcal{O}_{Y}, \mathcal{O}_{Z}\right)=I(Y, Z)
$$

in case that $Y \cap Z=\{0\}$. Here $I(Y, Z)$ is the ordinary intersection multiplicity of $Y$ and $Z$ in the ambient smooth space $\left(\mathbb{C}^{n+1}, 0\right)$.

Idea of proof: The result follows easily from Serre's Tor formula for the intersection multiplicity and the "change of rings exact sequence"

$$
\begin{aligned}
& \cdots \longrightarrow \operatorname{Tor}_{k}^{P}(M, N) \longrightarrow \operatorname{Tor}_{k}^{R}(M, N) \longrightarrow \operatorname{Tor}_{k-2}^{R}(M, N) \longrightarrow \operatorname{Tor}_{k-1}^{R}(M, N) \longrightarrow \operatorname{Tor}_{k-3}^{R}(M, N) \longrightarrow \\
& \longrightarrow
\end{aligned}
$$

relating Tor over $P$ and over $R$.

On the other hand, if $f \in P=\mathbb{C}\left[x_{1}, x_{2}, \ldots, x_{2 m+2}\right]$ is a homogeneous polynomial of degree $d$ in $2 m+2$ variables, then $f$ defines a homogeneous cone $X_{0}=f^{-1}(0) \subset$ $\mathbb{C}^{2 m+2}$ and an associated $2 m$-dimensional projective hypersurface

$$
T:=V(f) \subset \mathbb{P}^{2 m+1}
$$

of degree $d$. If $Y$ and $Z$ are homogeneous sub-varieties of $X_{0}$ of codimension $m$, then the projectivizations of $Y$ and $Z$ are codimension $m$ cycles in $T$, whose fundamental classes in $H^{m}(T)$ we denote by $[Y]$ and $[Z]$, respectively. The graded version of Hochster's theorem just stated then yields the following result.

Theorem 2.2. If $Y$ and $Z$ intersect transversely, then

$$
\theta\left(\mathcal{O}_{Y}, \mathcal{O}_{Z}\right)=-\frac{1}{d}[[Y]] \cdot[[Z]],
$$

where $[[Y]]:=d[Y]-\operatorname{deg}(Y) \cdot h^{m}$ is the primitive class of $[Y]$, with $h \in H^{1}(T)$ the hyperplane class, and $\operatorname{deg}(Y)$ the degree of the subvariety $Y$ in $\mathbb{P}^{2 m+1}$.

Proof: Recall that the primitive class of a cycle $Y$ is the projection of its fundamental class $[Y] \in H^{m}(T)$ into the orthogonal complement to $h^{m}$ with respect to the intersection pairing into $H^{2 m}(T) \cong \mathbb{C}$. As $h^{m} h^{m}=d=\operatorname{deg}(T)$ and $[Y] \cdot h^{m}=\operatorname{deg}(Y)$, the description $[[Y]]=d[Y]-\operatorname{deg}(Y) h^{m}$ of the primitive class follows. Substituting, the claim can be reformulated as

$$
\theta\left(\mathcal{O}_{Y}, \mathcal{O}_{Z}\right)=-\frac{1}{d}[[Y]] \cdot[[Z]]=-d[Y] \cdot[Z]+\operatorname{deg}(Y) \operatorname{deg}(Z),
$$


where $[Y] \cdot[Z]$ denotes the intersection form on the cohomology of projective space. The claim on Hochster's Theta pairing now follows from an argument on HilbertPoincaré series, the generating functions $\mathbb{H}(M)=\sum_{i} \operatorname{dim}\left(M_{i}\right) t^{i}$, where $M=$ $\oplus_{i \in \mathbb{Z}} M_{i}$ is a finitely generated graded module. For a complex of graded modules $C^{j}$ set $\mathbb{H}\left(C^{\bullet}\right)=\sum_{j}(-1)^{j} \mathbb{H}\left(C^{j}\right)$. The latter alternating sum is defined, as long as for a fixed degree $i$ only finitely many of the modules $C^{j}$ satisfy $C_{i}^{j} \neq 0$, and in that case $\mathbb{H}\left(C^{\bullet}\right)=\mathbb{H}\left(H\left(C^{\bullet}\right)\right)$.

If $C^{\bullet}=\left(\cdots C^{1} \rightarrow C^{0}\right) \rightarrow M$ is a minimal homogeneous free resolution, then its terms $C^{j}$ are generated in higher and higher degrees as $j$ increases, whence $\mathbb{H}\left(C^{\bullet}\right)$ is summable. Exactness shows the alternating sum to equal $\mathbb{H}(M)$.

If $N$ is a second finitely generated graded module and $D^{\bullet} \rightarrow N$ a minimal homogeneous free resolution, then the complex $C^{\bullet} \otimes_{R} D^{\bullet}$ that is the (total complex of the) tensor product of the resolutions still satisfies the summability condition. Its Hilbert-Poincaré series is independent of the choice of resolutions and denoted $\mathbb{H}\left(M \stackrel{\mathrm{E}}{\otimes}{ }_{R} N\right)$. It follows readily; see, for example, $[\mathrm{AvB}$, Lemma 7]; that

$$
\mathbb{H}\left(M \stackrel{\mathrm{E}}{\otimes_{R}} N\right)=\frac{\mathbb{H}(M) \mathbb{H}(N)}{\mathbb{H}(R)} .
$$

Furthermore, in case that $M=\mathcal{O}_{Y}, N=\mathcal{O}_{Z}$, for cycles $Y, Z$ that intersect transversely, $\operatorname{Tor}_{i}^{R}(M, N)$ is of finite length for $i>0$, and $\operatorname{Tor}_{i+2}^{R}(M, N)$ is isomorphic as graded vector space to $\operatorname{Tor}_{i}^{R}(M, N)$, shifted in degrees by $d$. Thus, equating the Hilbert-Poincaré series of $M \stackrel{\mathrm{E}}{\otimes}{ }_{R} N$ with that of its homology results in $\mathbb{H}\left(M \stackrel{\mathbb{L}}{\otimes_{R}} N\right)=\mathbb{H}\left(M \otimes_{R} N\right)+$ polynomial $+\frac{\mathbb{H}\left(\operatorname{Tor}_{e v}^{R}(M, N)\right)-\mathbb{H}\left(\operatorname{Tor}_{o d d}^{R}(M, N)\right)}{1-t^{d}}$.

Next observe that $M \otimes_{R} N \cong M \otimes_{P} N$ and that $\operatorname{Tor}_{i}^{P}(M, N)$ too is of finite length for $i>0$, equal to zero for $i>2 m+2$. Thus, $\mathbb{H}\left(M \otimes_{R} N\right)$ and $\mathbb{H}\left(M \stackrel{\mathrm{E}}{\otimes_{P}} N\right)$ differ only by a polynomial. Now compare the residues at $t=1$ :

Because $Y, Z$ are of codimension $m$ in $X_{0}$ of dimension $2 m+1$, the Hilbert-Poincaré series of $M, N$ are of the form

$$
\mathbb{H}(M)=\frac{p_{M}}{(1-t)^{m+1}}, \quad \mathbb{H}(N)=\frac{p_{N}}{(1-t)^{m+1}},
$$

with $p_{M}, p_{N} \in \mathbb{Z}[t]$ satisfying $p_{M}(1)=\operatorname{deg}(Y), p_{N}(1)=\operatorname{deg}(Z)$, whence

$$
\mathbb{H}\left(M \stackrel{\llcorner}{\otimes_{R}} N\right)=\frac{\mathbb{H}(M) \mathbb{H}(N)}{\mathbb{H}(R)}=\frac{p_{M} p_{N}}{1-t^{d}},
$$

as $\mathbb{H}(R)=\left(1-t^{d}\right) /(1-t)^{2 m+2}$. Therefore, the residue evaluates to

$$
\operatorname{res}_{t=1} \mathbb{H}\left(M \stackrel{\mathrm{Ł}}{\otimes_{R}} N\right)=\frac{1}{d} p_{M}(1) p_{N}(1)=\frac{1}{d} \operatorname{deg}(Y) \operatorname{deg}(Z) .
$$

For the Hilbert-Poincaré series $\mathbb{H}\left(M \otimes_{R} N\right)$ we get

$$
\operatorname{res}_{t=1} \mathbb{H}\left(M \otimes_{R} N\right)=\operatorname{res}_{t=1} \mathbb{H}\left(M \stackrel{\mathrm{E}}{\otimes}_{P} N\right)=[Y] \cdot[Z] .
$$

Finally,

$$
\operatorname{res}_{t=1} \frac{\mathbb{H}\left(\operatorname{Tor}_{e v}^{R}(M, N)\right)-\mathbb{H}\left(\operatorname{Tor}_{o d d}^{R}(M, N)\right)}{1-t^{d}}=\frac{1}{d} \theta\left(\mathcal{O}_{Y}, \mathcal{O}_{Z}\right)
$$


Putting it all together, the equality of residues becomes

$$
\frac{1}{d} \operatorname{deg}(Y) \operatorname{deg}(Z)=[Y] \cdot[Z]+\frac{1}{d} \theta\left(\mathcal{O}_{Y}, \mathcal{O}_{Z}\right)
$$

and solving for the Theta pairing yields the claim.

Example 2.3. Let $f \in \mathbb{C}\left[x_{1}, x_{2}, x_{3}, x_{4}\right]$ be the equation of a cubic surface $S$ in $\mathbb{P}^{3}$. $A$ line $L$ on $S$ is given by two linear forms $l_{1}, l_{2}$ such that $f=l_{1} q_{1}+l_{2} q_{2}$, for suitable quadratic polynomials $q_{1}, q_{2}$.

The matrix factorization associated to $L$ has the form

$$
A=\left(\begin{array}{cc}
l_{1} & -q_{2} \\
l_{2} & q_{1}
\end{array}\right), B=\left(\begin{array}{cc}
q_{1} & q_{2} \\
-l_{2} & l_{1}
\end{array}\right),
$$

and one easily determines from this $\theta\left(\mathcal{O}_{L}, \mathcal{O}_{L^{\prime}}\right)$ for a pair of lines on $S$. The Theta pairing then recovers the $E_{6}$-lattice on the primitive cohomology of a smooth cubic surface from the configuration of the lines on $S$. In fact, for lines $L, L^{\prime}$ one has the following table of dimensions of the torsion groups and values of Hochster's Theta pairing:

\begin{tabular}{|c|c|c|c|}
\hline position & skew & transverse & identical \\
\hline $\operatorname{Tor}_{\text {even }}^{R}\left(\mathcal{O}_{L}, \mathcal{O}_{L^{\prime}}\right)$ & 1 & 0 & 0 \\
$\operatorname{Tor}_{\text {odd }}^{R}\left(\mathcal{O}_{L}, \mathcal{O}_{L^{\prime}}\right)$ & 0 & 2 & 4 \\
\hline$\theta\left(\mathcal{O}_{L}, \mathcal{O}_{L^{\prime}}\right)$ & 1 & -2 & -4 \\
\hline
\end{tabular}

and in each case this agrees with the value predicted by the preceding result, even when the cycles are not transversal.

If, however, $f$ is not (quasi-) homogeneous, there no longer will be a projective variety to do intersection theory on. So the question arises as to the meaning of $\theta$ in the general case. For this, the geometry of the link $L=X_{0} \cap S$ of the isolated singularity inside the Milnor sphere will be relevant. We will continue with the case $n=2 m+2$. The non-vanishing cohomology groups of $L$ then are:

$$
\mathbb{Z}=H^{0}(L), \quad H^{2 m}(L), \quad H^{2 m+1}(L), \quad H^{4 m+1}(L)=\mathbb{Z} .
$$

The fundamental class of a codimension $m$ cycle on $X_{0}$ will land in $H^{2 m}(L)$, but if we want to attach a number to two classes $[A],[B] \in H^{2 m}(L)$ something new has to be involved, because the dual space to $H^{2 m}(L)$ is $H^{2 m+1}(L)$. J. Steenbrink came up with the following conjecture:

Conjecture 2.4. ( $\underline{\mathrm{St}})$ :

$$
\theta\left(\mathcal{O}_{A}, \mathcal{O}_{B}\right)=l k([A],[B]) .
$$

Here $l k: H^{2 m}(L) \times H^{2 m}(L) \longrightarrow \mathbb{Z}$ is the co-called linking form, while $[A] \in H^{2 m}(L)$ is the topological fundamental class obtained by intersecting the cycle $A$ on $X_{0}$ with the Milnor sphere $S$.

The formula states that, geometrically, one takes the classes $[A]$ and $[B]$, then shifts $[A]$ transverse to $L$ into $S=S^{4 m+3}$ to get $\widetilde{[A]}$. The cycles $\widetilde{[A]}$ and $[B]$ now have the 
right codimensions to link in $S$. To shift, the canonical trivialization of the normal bundle to $L$ determined by the values of the function $f$ is used.

Example 2.5. Let us consider the earlier example, where $M=\mathbb{C}\{x, y\} /(x)$, $N=\mathbb{C}\{x, y\} /(y)$ on the $A_{1}$ singularity $x y=0$. The classes $[M]$ and $[N]$ are geometrically represented by circles $x=0,|y|=\epsilon$ and $y=0,|x|=\epsilon$ respectively, with their standard anti-clockwise orientation. The linking number of these circles is +1 . The cycle $\widetilde{[M]}$ is represented by the circle $x y=t$, for $|t| \neq 0$ small and fixed, $|y|$ fixed. We see that if $y$ runs anti-clockwise, then $x$ runs clockwise, so the linking number of $\widetilde{[M]}$ with $[N]$ is -1 . This is in accordance with the computation of Hochster's Theta pairing.

Originally, J. Steenbrink formulated the conjecture in terms of the variation mapping in the Milnor fibration, but this is equivalent to the above conjecture by standard results on the topology of isolated hypersurface singularities, see section 5 . We note that the conjecture is compatible with the special cases covered by theorem 1 and 2. The following is the main result of this paper:

Main Theorem Let $f \in P:=\mathbb{C}\left\{x_{0}, x_{1}, \ldots, x_{n}\right\}$ define an isolated singularity, and let $M$ and $N$ be $R=P /(f)$-modules.

(i) If $n$ is odd, then $\theta(M, N)=0$.

(ii) If $n$ is even, then

$$
\theta(M, N)=l k(\operatorname{ch}(M), \operatorname{ch}(N)) .
$$

Here ch: $K^{0}(L) \longrightarrow H^{e v}(L)$ is the Chern-character. Only the $2 m$-component $c h^{2 m} \in H^{2 m}(L ; \mathbb{Q})$ contributes to the linking, so that alternatively we might write $\theta(M, N)=l k\left(c h^{2 m}(M), c h^{2 m}(N)\right)$.

\section{Interpretation in Algebraic K-Theory}

We start with a description of $\theta(M, N)$ in terms of algebraic $K$-theory. After Quillen, K-theory can be defined for any abelian (or even exact) category $\mathcal{A}$. In either case, $K_{0}(\mathcal{A})$ is just the Grothendieck group of $\mathcal{A}$, so elements are represented as formal differences $[X]-[Y]$ of isomorphism classes of objects in $\mathcal{A}$ modulo relations $0=[X]-[Y]+[Z]$ for each exact sequence $0 \longrightarrow X \longrightarrow Y \longrightarrow Z \longrightarrow 0$.

A fundamental result obtained by Quillen $\mathrm{Q}$ is the localization sequence: a Serresubcategory $\mathcal{S}$ of $\mathcal{A}$ gives rise to a long exact sequence of higher $K$-groups

$$
\cdots \longrightarrow K_{i}(\mathcal{S}) \longrightarrow K_{i}(\mathcal{A}) \longrightarrow K_{i}(\mathcal{A} / \mathcal{S}) \stackrel{\partial}{\longrightarrow} K_{i-1}(\mathcal{S}) \longrightarrow K_{i-1}(\mathcal{A}) \longrightarrow \cdots
$$

Elements in higher $K$-groups are harder to describe. Gillet-Grayson GG], identify the higher groups as $K_{i}(\mathcal{A})=\pi_{i}(G \mathcal{A})$, where $G \mathcal{A}$ is a certain simplicial space, whose $k$-simplices are given by pairs of flags of objects in $\mathcal{A}$

$$
\begin{gathered}
X_{0} \hookrightarrow X_{1} \hookrightarrow \cdots \hookrightarrow X_{k} \\
Y_{0} \hookrightarrow Y_{1} \hookrightarrow \cdots \hookrightarrow Y_{k}
\end{gathered}
$$

with compatible identifications $X_{j} / X_{i} \approx Y_{j} / Y_{i}$. Nenashev [N] has shown, building on earlier work of Sherman Sh1], that all elements in $K_{1}(\mathcal{A})$ can be represented by 
so-called double short exact sequences (d.s.e.s): these are pairs of exact sequences on the same three objects of $\mathcal{A}$ :

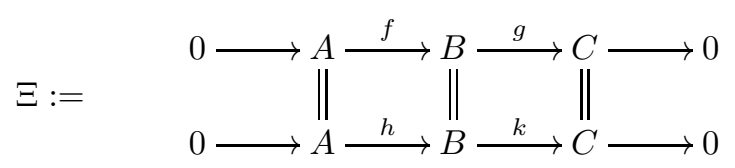

(Of course, the diagrams are not supposed to commute.)

Examples 3.1. (i) The elements where $A=0$ lead to special $K_{1}$-elements, associated to a pair of isomorphisms, or to an automorphism of a single object. In the set-up of $[\mathrm{N}]$, an automorphism $\beta: B \longrightarrow B$ corresponds to the d.s.e.s

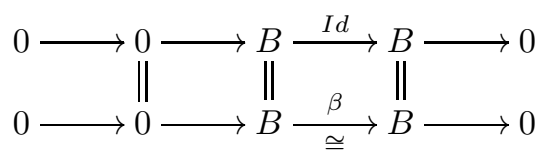

(ii) By a cyclic diagram in $\mathcal{A}$ we mean a diagran 2 of the form

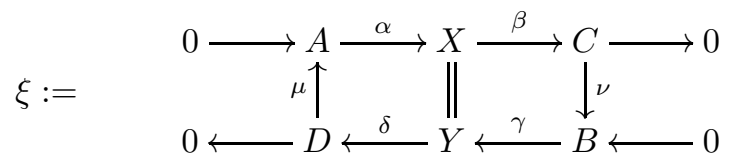

where both rows are short exact sequences in $\mathcal{A}$. In case that $\mu$ and $\nu$ are isomorphisms, such a diagram determines a class $\{\xi\} \in K_{1}(\mathcal{A})$. As some maps are going in the wrong direction, we do not have a d.s.e.s, but one can obtain one by considering the diagram

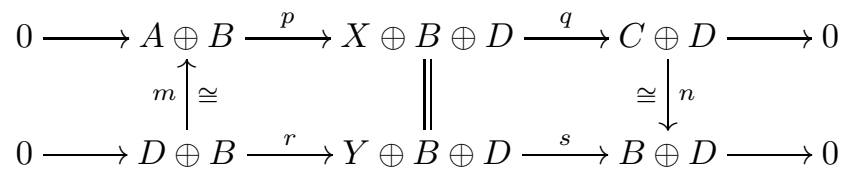

where the maps are in block-form, defined as follows

$$
p:=\left(\begin{array}{cc}
\alpha & 0 \\
0 & 1 \\
0 & 0
\end{array}\right), \quad q:=\left(\begin{array}{ccc}
\beta & 0 & 0 \\
0 & 0 & 1
\end{array}\right), \quad r:=\left(\begin{array}{cc}
0 & \gamma \\
0 & 0 \\
1 & 0
\end{array}\right), \quad s:=\left(\begin{array}{ccc}
0 & 1 & 0 \\
\delta & 0 & 0
\end{array}\right)
$$

and $m:=\left(\begin{array}{cc}\mu & 0 \\ 0 & 1\end{array}\right), n=\left(\begin{array}{cc}\nu & 0 \\ 0 & 1\end{array}\right)$. Thus we get a d.s.e.s

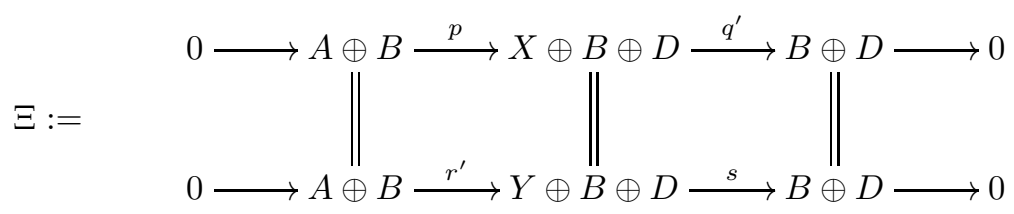

where $q^{\prime}:=n q, r^{\prime}:=r m^{-1}$. So it determines a class

$$
\{\xi\}:=[\Xi] \in K_{1}(\mathcal{A}) .
$$

\footnotetext{
${ }^{2}$ Although $X=Y$, we prefer to distinguish these two copies of the same object to indicate clearly in the following diagrams, where each copy comes from.
} 
(iii) A 2-periodic complex $C^{\bullet}$ in $\mathcal{A}$ :

$$
\stackrel{a}{\longrightarrow} Y \stackrel{b}{\longrightarrow} X \stackrel{a}{\longrightarrow} Y \stackrel{b}{\longrightarrow} X \stackrel{a}{\longrightarrow} \cdots
$$

(where $Y$ is on the even spots and $X=Y$ ) determines a canonical cyclic diagram

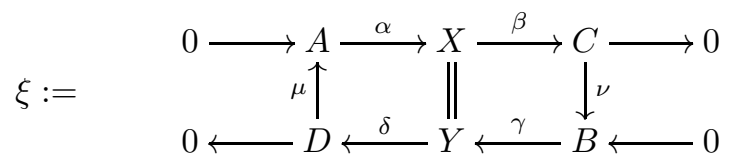

where $A=\operatorname{Ker}(a), C=\operatorname{Im}(a), B=\operatorname{Ker}(b), D=\operatorname{Im}(b)$.

If the complex is exact, then $\mu$ and $\nu$ are isomorphisms, and so $C^{\bullet}$ determines the class

$$
\left\{C^{\bullet}\right\}:=\{\xi\} \in K_{1}(\mathcal{A})
$$

The boundary map. Sherman Sh2 has given an explicit description for the boundary map

$$
K_{1}(\mathcal{A}) \stackrel{\partial}{\longrightarrow} K_{0}(\mathcal{S})
$$

that we will need. If $f: A \longrightarrow B$ is a morphism in $\mathcal{A}$, let us denote, as in Sh2, p.177], by an overline the corresponding map in $\mathcal{A} / \mathcal{S}: \bar{f}: \bar{A} \longrightarrow \bar{B}$.

If $f: A \longrightarrow B$ in $\mathcal{A}$ is an $\mathcal{S}$-isomorphism, that is, $\operatorname{Ker}(f), \operatorname{Coker}(f) \in \mathcal{S}$, we put

$$
\chi(f):=[\operatorname{Coker}(f)]-[\operatorname{Ker}(f)] \in K_{0}(\mathcal{S})
$$

If $A, B$ in $\mathcal{A}$ are lifts of $\bar{A}, \bar{B}$ in $\mathcal{A} / \mathcal{S}$ and $\phi: \bar{A} \longrightarrow \bar{B}$ is an $\mathcal{A} / \mathcal{S}$-morphism, then it can be "lifted" to a diagram

$$
A \stackrel{a}{\longleftarrow} C \stackrel{f}{\longrightarrow} D \stackrel{b}{\longleftarrow} B
$$

where $a$ and $b$ are $\mathcal{S}$-isomorphisms and $\phi=\bar{b}^{-1} \bar{f} \bar{a}^{-1}$. In case $f$ itself is an $\mathcal{S}$ isomorphism, one puts

$$
\chi(\phi):=\chi(f)-\chi(a)-\chi(b) \in K_{0}(\mathcal{S}) .
$$

This class is independent of the choices made.

Given an element $x \in K_{1}(\mathcal{A} / \mathcal{S})$ represented by a double short exact sequence

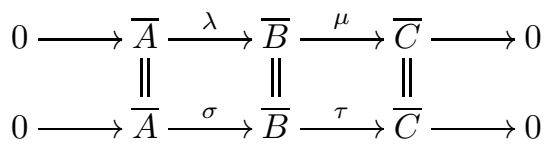


one can "lift" the morphisms involved in such a way ${ }^{3}$ as to obtain an extended diagram in $\mathcal{A} / \mathcal{S}$ with exact rows and commuting squares at the top and bottom,

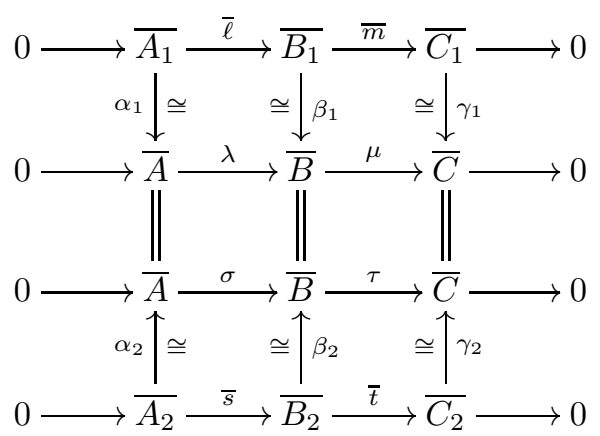

Theorem 3.2. (Theorem 2.3 of [Sh2])

The boundary map $K_{1}(\mathcal{A} / \mathcal{S}) \stackrel{\partial}{\longrightarrow} K_{0}(\mathcal{S})$ sends the element $x$ to

$$
\partial(x)=\chi(\alpha)-\chi(\beta)+\chi(\gamma) \in K_{0}(\mathcal{S})
$$

where

$$
\alpha=\alpha_{2}^{-1} \alpha_{1}, \quad \beta=\beta_{2}^{-1} \beta_{1}, \quad \gamma=\gamma_{2}^{-1} \gamma_{1}
$$

Corollary 3.3. If in a cyclic diagram

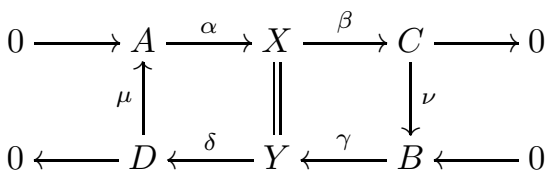

in $\mathcal{A}$ the morphisms $\mu, \nu$ are $\mathcal{S}$-isomorphisms, then the reduction

$$
\begin{array}{cc}
0 \longrightarrow \bar{A} \stackrel{\bar{\alpha}}{\longrightarrow} \bar{X} \stackrel{\bar{\beta}}{\longrightarrow} \bar{C} \longrightarrow 0 \\
\bar{\mu} \uparrow \cong \quad \cong \mid \bar{\nu} \\
0 \longleftarrow \bar{D} \stackrel{\bar{\delta}}{\longleftarrow} \bar{Y} \stackrel{\bar{\gamma}}{\longleftarrow} \bar{B} \longleftarrow 0
\end{array}
$$

in $\mathcal{A} / \mathcal{S}$ satisfies:

$$
\partial(\{\xi\})=([\operatorname{Coker}(\nu)]-[\operatorname{Ker}(\nu)])-([\operatorname{Coker}(\mu)]-[\operatorname{Ker}(\mu)]) .
$$

Proof: The d.s.e.s associated to $\xi$ comes with given lifts to $\mathcal{A}$ from the cyclic diagram in $\mathcal{A}$. The result now follows by a direct application of Sherman's theorem to the associated double short exact sequence.

Construction for hypersurfaces. We will apply the above theory to our situation of modules on a hypersurface ring $R$ with an isolated singular point. We let $\mathcal{A}:=\bmod (R)$, the category of finitely generated $R$-modules and $\mathcal{S}=\operatorname{art}(R)$, the Serre-subcategory of artinian $R$-modules, or what is the same, of those that are supported at the singular point. The category $\mathcal{A} / \mathcal{S}$ is the category obtained by "localizing away" from the singular point and can be identified with the category $\operatorname{coh}(U)$ of coherent sheaves on the punctured spectrum $U:=X_{0} \backslash\{0\}$. We will write $\mathbf{K}^{i}(U)$ for $K_{i}(\operatorname{coh}(U))$. As $U$ is smooth, it is the same as $K_{i}(\operatorname{Vect}(U))$, where

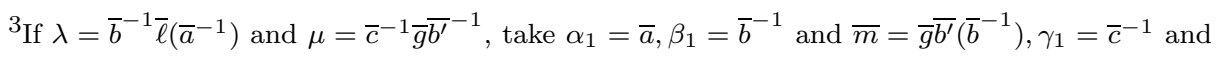
so on.
} 
$\operatorname{Vect}(U)$ is the category of locally free $\mathcal{O}_{U}$-modules.

Definition: For a maximal Cohen-Macaulay module $M$ on a hypersurface ring $R$ we put:

$$
\begin{gathered}
{[M]=\left[M_{U}\right] \in \mathbf{K}^{0}(U)} \\
\{M\}:=\left\{C^{\bullet}(M)_{U}\right\} \in \mathbf{K}^{1}(U)
\end{gathered}
$$

where $(.)_{U}$ denotes restriction to $U$.

We recall that there exists a natural product

$$
\mathbf{K}^{1}(U) \times \mathbf{K}^{0}(U) \longrightarrow \mathbf{K}^{1}(U)
$$

induced in the obvious way by the tensor product $\otimes$ of modules, and a trace map

$$
\chi:=\ell \circ \partial: \mathbf{K}^{1}(U) \longrightarrow \mathbb{Z}
$$

that is the composition of the boundary map $\mathbf{K}^{1}(U) \stackrel{\partial}{\longrightarrow} K_{0}(\operatorname{art}(R))$ and the isomorphism $\ell: K_{0}(\operatorname{art}(R)) \longrightarrow \mathbb{Z}, \quad M \mapsto \operatorname{length}(M)$.

The following crucial result expresses Hochster's Theta pairing in terms of algebraic $K$-theory.

\section{Theorem 3.4.}

$$
\theta(M, N)=\chi(\{M\} \otimes[N])
$$

Proof: Recall the exact 2-periodic complex of $R$-modules

$$
C^{\bullet}(M): \quad \cdots \stackrel{B}{\longrightarrow} G \stackrel{A}{\longrightarrow} F \stackrel{B}{\longrightarrow} G \stackrel{A}{\longrightarrow} F \stackrel{B}{\longrightarrow} \cdots
$$

that is defined by (a matrix factorisation $(A, B)$ attached to) $M$. By definition, the groups $\operatorname{Tor}_{\text {even }}^{R}$ odd $(M, N)$ are obtained by tensoring this complex with $N$ and then taking homology. To put this differently, consider the following cyclic diagram

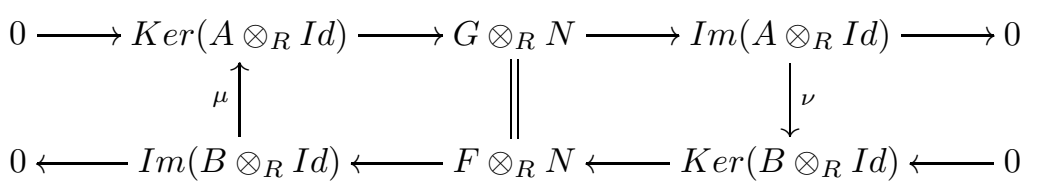

where $I d: N \longrightarrow N$ is the identity map. We then have

$$
\operatorname{Tor}_{\text {even }}^{R}(M, N)=\operatorname{Coker}(\nu), \operatorname{Tor}_{\text {odd }}^{R}(M, N)=\operatorname{Coker}(\mu) .
$$

On the other hand, the class $\{M\} \in \mathbf{K}^{1}(U)$ is represented by the cyclic diagram and resulting d.s.e.s. associated to the exact 2-periodic complex

$$
\overline{C^{\bullet}(M)}: \quad \cdots \stackrel{\bar{B}}{\longrightarrow} \bar{G} \stackrel{\bar{A}}{\longrightarrow} \bar{F} \stackrel{\bar{B}}{\longrightarrow} \bar{G} \stackrel{\bar{A}}{\longrightarrow} \bar{F} \stackrel{\bar{B}}{\longrightarrow} \cdots
$$

where the overline indicates the restriction to $U$. As we are now outside the singular locus, the tensor product

$$
\cdots \longrightarrow \bar{G} \otimes \bar{N} \stackrel{\bar{A} \otimes \overline{I d}}{\longrightarrow} \bar{F} \otimes \bar{N} \stackrel{\bar{B} \otimes \overline{I d}}{\longrightarrow} \bar{G} \otimes \bar{N} \stackrel{\bar{A} \otimes \overline{I d}}{\longrightarrow} \bar{F} \otimes \bar{N} \stackrel{\bar{B} \otimes \overline{I d}}{\longrightarrow} \cdots
$$


of $\overline{C^{\bullet}(M)}$ with $\bar{N}$ on $U$ stays exact, and the class $\{M\} \otimes[N] \in \mathbf{K}^{1}(U)$ is represented by the cyclic diagram

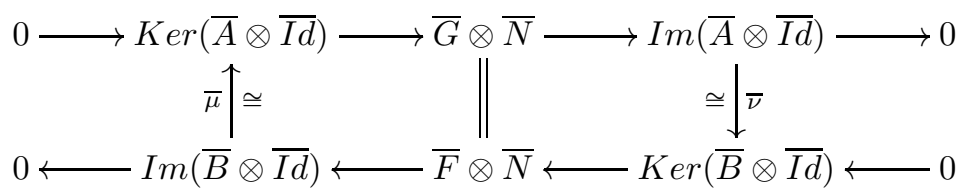

associated to that tensor product on $U$.

It follows now from the corollary to the theorem of Sherman that

$$
\begin{aligned}
\partial(\{M\} \otimes[N]) & =[\operatorname{Coker}(\nu)]-[\operatorname{Coker}(\mu)] \\
& =\left[\operatorname{Tor}_{\text {even }}^{R}(M, N)\right]-\left[\operatorname{Tor}_{\text {odd }}^{R}(M, N)\right]
\end{aligned}
$$

and applying the trace map completes the argument.

\section{INTERPRETATION IN TERMS OF TOPOLOGICAL K-THEORY}

Topological $K$-theory is a generalized cohomology theory obtained from topological $\mathbb{C}$-vector bundles. We refer to Atiyah's classic [A] for a nice introduction. This theory fits into the above framework, if we take for any topological space $X$ the abelian category $V e c t_{\text {top }}(X)$ of topological $\mathbb{C}$-vector bundles on it:

$$
K^{i}(X)=K_{i}\left(\operatorname{Vect}_{t o p}(X)\right) .
$$

As vector bundles can be pulled back, this is naturally a contravariant functor. Distinctive features of the theory are:

- In $V e c t_{t o p}(X)$ all (short) exact sequences can be split: if $E \stackrel{i}{\hookrightarrow} F \rightarrow G$ is such a sequence, just pick a hermitian metric on $F$ and use orthogonal projection to obtain $F \cong E \oplus E^{\perp}, E^{\perp} \cong G$.

- One has Bott periodicity: $K^{i}(X) \cong K^{i+2}(X)$. So we really have to consider only the two groups $K^{0}$ and $K^{1}$.

- Elements of $K^{1}$ can all be represented by automorphisms $E \stackrel{\alpha}{\longrightarrow} E$ of a vector bundle on $X$. If $x=[E \stackrel{\alpha}{\longrightarrow} E]$, then $-x=\left[E \stackrel{\alpha^{-1}}{\longrightarrow} E\right]$.

- The elements in $K^{1}$ are homotopy invariants: if $t \mapsto \alpha_{t}$ is a path within the space of automorphisms of $E$, then $x_{t}=\left[E \stackrel{\alpha_{t}}{\longrightarrow} E\right]$ is independent of $t$.

- There are Chern-class maps

$$
\begin{gathered}
c h^{e v}: K^{0}(X) \longrightarrow H^{e v}(X)=\bigoplus_{k} H^{2 k}(X, \mathbb{Q}) \\
c h^{\text {odd }}: K^{1}(X) \longrightarrow H^{\text {odd }}(X)=\bigoplus_{k} H^{2 k+1}(X, \mathbb{Q})
\end{gathered}
$$

that are isomorphisms after tensoring with $\mathbb{Q}$.

- There is a version of Riemann-Roch in the differentiable context, stating that for a proper map, direct image in K-theory (defined via duality), commutes with taking Chern-classes, up to multiplication with the Todd-class of the virtual tangent bundle of the map.

Of importance for us will be the special case of the constant map $p: L \longrightarrow$ point, where $L$ is the link, a compact, odd-dimensional manifold with stably trivial tangent bundle. The induced map

$$
\chi_{\text {top }}:=p_{*}: K^{1}(L) \longrightarrow K^{0}(\text { point })=\mathbb{Z}
$$


is the trace map in topological $K$-theory.

As $U$ and $L$ are homotopy equivalent, we will identify $K^{i}(U)$ with $K^{i}(L)$ without further mention, and write also $\chi_{\text {top }}: K^{1}(U) \longrightarrow \mathbb{Z}$, and so on.

We can compare algebraic and topological $K$-theory for $U$ using the obvious topologification functor

$$
\text { top }: \operatorname{Vect}(U) \longrightarrow V e c t_{t o p}(U)
$$

sending a locally free sheaf to its associated topological vector bundle. This induces natural maps from algebraic to topological $K$-theory,

$$
\begin{aligned}
& \mathbf{K}^{0}(U) \quad \longrightarrow \quad K^{0}(U)=K^{0}(L) \quad, \quad[M] \quad \mapsto \quad[M]_{t o p}, \\
& \mathbf{K}^{1}(U) \longrightarrow K^{1}(U)=K^{1}(L) \quad,\{M\} \quad \mapsto\{M\}_{\text {top }} .
\end{aligned}
$$

\section{Proposition 4.1.}

$$
\theta(M, N)=\chi_{t o p}\left(\{M\}_{t o p} \otimes[N]_{t o p}\right)
$$

Proof: This follows from the naturality of topologification: $(\{M\} \otimes[N])_{t o p}=$ $\{M\}_{\text {top }} \otimes[N]_{\text {top }}$, and compatibility of $\chi$ and $\chi_{\text {top }}$, that is, the commutativity of

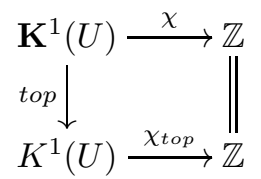

This "well-known" fact can be shown as follows. Take a finite map $p: X_{0} \longrightarrow Z$, where $Z$ is smooth and contractible. We let $Z^{*}=Z \backslash\{0\}$, so that $p: U \longrightarrow Z^{*}$ is a finite (ramified) covering. By functoriality of both $\mathbf{K}^{1}$ and $K^{1}$ and the fact that the map to the point factors over $p$, we reduce to checking the statement for $Z^{*}$. The structure sheaf of the point $\{0\}$ is resolved by the Koszul complex; its class in either $\mathbf{K}^{1}(U)$ or $K^{1}(U)$ is mapped by $\chi$, respectively $\chi_{\text {top }}$, to $1 \in \mathbb{Z}$.

A different description of $\{M\}_{t o p}$. If $(A, B)$ is a matrix factorisation for a maximal Cohen-Macaulay module $M$ on $X_{0}$, we can choose our good representative for $f: X \longrightarrow D$ in such a way that the matrices $A$ and $B$ are holomorphic on $X$. Hence we have an exact sequence of sheaves on $X$ :

$$
0 \longrightarrow \mathcal{O}_{X}^{p} \stackrel{A}{\longrightarrow} \mathcal{O}_{X}^{p} \longrightarrow M \longrightarrow 0 \text {. }
$$

(We will not make a notational distinction between objects over the local ring and corresponding sheaves on $X)$. As $\operatorname{det}(A)$ vanishes only on the hypersurface $X_{0}$, the matrix $A(x)$ is an isomorphism for each $x \in X^{*}=X \backslash X_{0}$. It determines hence a class

$$
\alpha(M):=\left[\mathcal{O}_{X^{*}}^{p} \stackrel{A}{\longrightarrow} \mathcal{O}_{X^{*}}^{p}\right] \in K^{1}\left(X^{*}\right)
$$

and, as $X^{*}$ retracts to $S-L$, we can also see $\alpha(M) \in K^{1}(S-L)=K^{1}\left(X^{*}\right)$. For a Milnor fibre $X_{t}$, we have inclusion maps $\partial X_{t} \hookrightarrow X_{t} \hookrightarrow X^{*}$, hence we get corresponding restriction maps

$$
K^{1}\left(X^{*}\right) \longrightarrow K^{1}\left(X_{t}\right) \longrightarrow K^{1}\left(\partial X_{t}\right)
$$


that send $\alpha(M)$ to $\left.\alpha(M)\right|_{X_{t}}$ and $\left.\alpha(M)\right|_{\partial X_{t}}$ respectively. Furthermore, the local triviality of the Milnor fibration near the boundary provides an identification $\rho_{t}: L \longrightarrow \partial X_{t}$, in particular, an isomorphism $\rho_{t}^{*}: K^{1}\left(\partial X_{t}\right) \longrightarrow K^{1}(L)$.

Theorem 4.2. For any $t \in D^{*}$, we have

$$
\{M\}_{\text {top }}=\rho_{t}^{*}\left(\left.\alpha(M)\right|_{\partial X_{t}}\right) .
$$

The proof will make use of the following lemma.

Lemma 4.3. Choose a hermitian metric $(-,-)$ on $\mathcal{O}_{X}^{p}$ and let $B^{\dagger}$ be the hermitian adjoint of $B$. For $s \in \mathbb{C}^{*}$, consider the matrix

$$
A_{s}:=A-s B^{\dagger} \text {. }
$$

(i) The matrix $A_{s}(x)$ is invertible for all $x \in X_{t}$ if $t \notin \mathbb{R}_{+} \cdot s$, so defines a class $\left.\alpha_{s}(M)\right|_{X_{t}} \in K^{1}\left(X_{t}\right)$, and that class satisfies

$$
\left.\alpha_{s}(M)\right|_{X_{t}}=\left.\alpha(M)\right|_{X_{t}} .
$$

(ii) For each $s \neq 0$, the matrix $A_{s}(x)$ is invertible on $U$, so defines a class $\alpha_{s}(M)_{L} \in$ $K^{1}(L)$. That class satisfies

$$
\alpha_{s}(M)_{L}=\rho_{t}^{*}\left(\left.\alpha(M)\right|_{\partial X_{t}}\right)
$$

for $t \notin \mathbb{R}_{+} \cdot s$.

Proof: (i) Set $V_{s}:=\left\{x \in X \mid \operatorname{det}\left(A_{s}(x)\right)=0\right\}$. If $x \in V_{s}$, then there exists by definition a vector $v=v(x) \neq 0$ in the kernel of $A_{s}(x)$. Hence, we have:

$$
A(x) \cdot v=s \cdot B^{\dagger}(x) \cdot v \text {. }
$$

When we multiply this equation from the left with $B(x)$, we get

$$
s^{-1} f(x) \cdot v=B(x) \cdot B^{\dagger}(x) \cdot v,
$$

that is, $s^{-1} f(x)$ is an eigenvalue of the matrix $B(x) \cdot B^{\dagger}(x)$. However, if $B(x) \cdot B^{\dagger}(x) v=$ $\lambda . v$, then $\left(B^{\dagger}(x) v, B^{\dagger}(x) v\right)=\lambda .(v, v)$, so $\lambda \geq 0$. It follows that the image under $f$ of $V_{s}$ is contained in the half-line $\mathbb{R}_{+} \cdot s$ and consequently $V_{s}$ is disjoint from the Milnor fibre $X_{t}$ if $t \notin \mathbb{R}_{+} \cdot s$. Note that $X_{t}$ is then also disjoint from $V_{s^{\prime}}$ for all $s^{\prime} \in \mathbb{R}^{+} s$. So $s^{\prime} \mapsto A_{s^{\prime}}$ provides a continuous path from $A=A_{0}$ to $A_{s}$ inside the space of invertible matrices on the Milnor fibre $X_{t}$. Hence, $A$ and $A_{s}$ represent the same element in $K^{1}\left(X_{t}\right)$.

(ii) If $x \neq 0$, then the eigenvalue $\lambda=0$ cannot occur for $B(x) \cdot B^{\dagger}(x)$ : first, if $\lambda=0$ then $B^{\dagger}(x) v=0$, and hence also $A(x) \cdot v=0$, as $v$ is in the kernel of $A_{s}(x)$. But then $0=\left(B^{\dagger}(x) v, w\right)=(v, B(x) w)$ shows that $v$ is orthogonal to $\operatorname{Im}(B(x))=\operatorname{Ker}(A(x))$, the last equality due to $x \in U$. As $v \in K \operatorname{Ker}(A(x))$, this shows $v=0$. We conclude that $A_{s}(x)$ is invertible for all $x \in U$ and thus defines by restriction a class $\alpha_{s}(M)_{L}$. By construction, $\alpha_{s}(M)_{L}=\rho_{t}^{*}\left(\left.\alpha_{s}(M)\right|_{\partial X_{t}}\right)$, so the last statement follows from (i).

Proof of the Theorem:

We look at the exact 2-periodic complex $C^{\bullet}(M)_{U}$ on $U$ :

$$
\ldots \stackrel{B}{\longrightarrow} G \stackrel{A}{\longrightarrow} F \stackrel{B}{\longrightarrow} G \stackrel{A}{\longrightarrow} \cdots
$$


where $F=G=\mathcal{O}^{p}$ is the trivial rank $p$ bundle on $U$ and $F$ sits on the even spots. In order to keep the notation simple, we will omit the overline or notation $(-)_{U}$ to denote restriction to $U$; everything here takes place in $V e c t_{t o p}(U)$. We choose a hermitian metric on $F$ and split the exact sequences

$$
\operatorname{Ker}(A) \hookrightarrow G \rightarrow G / \operatorname{Ker}(A), \quad \operatorname{Ker}(B) \hookrightarrow F \rightarrow F / \operatorname{Ker}(B),
$$

to obtain isomorphisms

$$
G \cong \operatorname{Ker}(A) \oplus G / \operatorname{Ker}(A), \quad F \cong F / \operatorname{Ker}(B) \oplus \operatorname{Ker}(B),
$$

that then give rise to a diagram

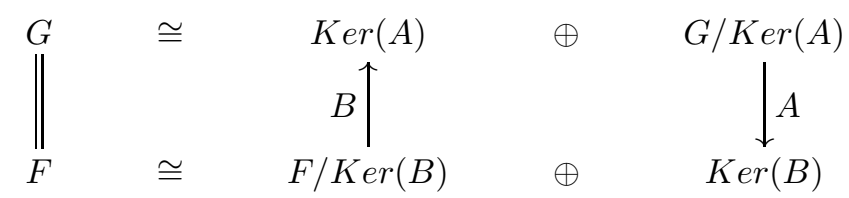

The class $\{M\}_{\text {top }}$ is represented by the automorphism $G \longrightarrow F$ obtained from this diagram by inverting the map $B$. Now, in general, if $E \stackrel{\alpha}{\longrightarrow} F$ is an isomorphism of vector bundles, and if $F \stackrel{\alpha^{\dagger}}{\longrightarrow} E$ is its hermitian adjoint, then $\alpha^{\dagger}$ is homotopic to $\alpha^{-1}$, as the composition $\alpha \alpha^{\dagger}$ is positive definite, hence homotopic to the identity. Accordingly our class $\{M\}_{\text {top }}$ is represented by the automorphism $A+B^{\dagger}$. But this is precisely $\alpha_{-1}(M)_{L}$, and by part (ii) of the lemma it represents the same class as $\rho_{t}^{*}\left(\left.\alpha(M)\right|_{\partial X_{t}}\right)$.

Corollary 4.4. If $n$ is even then $\theta(M, N)=0$ for all $M$ and $N$.

Proof: If $n$ is even, then $K^{1}\left(X_{t}\right)=0$, as $X_{t}$ has the homotopy type of a wedge of even dimensional spheres. Hence, the class of $M$ trivially satisfies $\left.\alpha(M)\right|_{X_{t}}=0$ in $K^{1}\left(X_{t}\right)$, and so certainly its restriction to the boundary vanishes also: $\left.\alpha(M)\right|_{\partial X_{t}}=$ 0 . But then

$$
\begin{aligned}
& \{M\}_{t o p}=\alpha_{s}(M)_{L}=\rho_{t}^{*}\left(\left.\alpha(M)\right|_{\partial X_{t}}\right)=0, \\
& \text { so } \theta(M, N)=\chi_{\text {top }}\left(\{M\}_{\text {top }} \otimes[N]_{t o p}\right)=0 !
\end{aligned}
$$

\section{The Linking Form}

We review here the basic properties of the linking pairing on the (co-)homology of the link of an isolated hypersurface singularity. More details can be found in AGV], Le].

Given two disjoint $n$-dimensional cycles $\alpha, \beta$ in the Milnor sphere $S=S^{2 n+1}$, we can form the linking number $\ell(\alpha, \beta) \in \mathbb{Z}$, which is defined as the intersection number $\Gamma \cdot \beta$ between a chain $\Gamma$ with $\partial \Gamma=\alpha$ and $\beta$. One has $\ell(\alpha, \beta)=(-1)^{n+1} \ell(\beta, \alpha)$, so that linking is symmetric for odd dimensional cycles. Consider the Milnor fibration $f: X^{*} \longrightarrow D^{*}$ as before. Fix $t \in D^{*}$ and use parallel transport along an anticlockwise half-turn from $t$ to $-t$ to define a "half-monodromy map"

$$
h_{1 / 2}: H_{n}\left(X_{t}\right) \longrightarrow H_{n}\left(X_{-t}\right) .
$$

If $\alpha, \beta \in H_{n}\left(X_{t}\right)$, then the cycles $\alpha$ and $h_{1 / 2}(\beta)$ are disjoint and have the appropriate dimension to link in the $2 n+1$ dimensional Milnor sphere $S$. The resulting Seifert form of the singularity is

$$
S: H_{n}\left(X_{t}\right) \times H_{n}\left(X_{t}\right) \longrightarrow \mathbb{Z}, \quad(\alpha, \beta) \mapsto \ell\left(\alpha, h_{1 / 2}(\beta)\right)
$$


If we restrict the Seifert form $S$ to $H_{n}(L) \cong H_{n}\left(\partial X_{t}\right) \subset H_{n}\left(X_{t}\right)$ we obtain a $(-1)^{n+1}$-symmetric form

$$
l k: H_{n}(L) \times H_{n}(L) \longrightarrow \mathbb{Z}
$$

that we call the linking form of the link. So, geometrically, $l k(\alpha, \beta)=\ell(\tilde{\alpha}, \tilde{\beta})$, where $\tilde{\alpha}$ and $\tilde{\beta}$ are obtained from $\alpha$ and $\beta$ using the identification $H_{n}(L) \approx H_{n}\left(\partial X_{t}\right)$ and $H_{n}(L) \approx H_{n}\left(\partial X_{-t}\right)$ respectively. Alternatively, we may say that $\tilde{\alpha}$ and $\tilde{\beta}$ are obtained by "pushing-aside" $\alpha$ and $\beta$ in opposite directions, using the trivialisation of the normal bundle of $L$ defined by $f$. It is clear that in fact one only needs to push aside one of the cycles, so that $l k(\alpha, \beta)=\ell(\tilde{\alpha}, \beta)$.

The geometric monodromy can be taken to be the identity on $\partial X_{t}$, so one can define a variation mapping

$$
\text { Var : } H_{n}\left(X_{t}, \partial X_{t}\right) \longrightarrow H_{n}\left(X_{t}\right)
$$

obtained by mapping a relative cycle $\gamma$ to $[\gamma-h(\gamma)]$, where $h$ is the monodromy. It is related to the Seifert form and the intersection pairing

$$
(-,-): H_{n}\left(X_{t}, \partial X_{t}\right) \times H_{n}\left(X_{t}\right) \longrightarrow \mathbb{Z}
$$

by the formula $S(\operatorname{Var}(\alpha), \beta)=(\alpha, \beta)$, and as the variation is an isomorphism, we can write $S(\alpha, \beta)=\left(\operatorname{Var}^{-1} \alpha, \beta\right)$.

Formulation in cohomology and K-theory. We will reformulate the above procedure in cohomological terms and cover it through a description in topological $K$-theory. We will restrict to the case $n$ odd, so that the form $l k$ is symmetric. We write $n+1=2 m+2$, so that the non-vanishing cohomology of $L$ sits in degrees $0,2 m, 2 m+1,4 m+1$, and $S$ has dimension $4 m+3$. Written in cohomology, the linking form becomes a pairing on the cohomology in degree $2 m$ :

$$
l k: H^{2 m}(L) \times H^{2 m}(L) \longrightarrow \mathbb{Z} .
$$

From the long exact cohomology sequence of the pair $(S, S-L)$ and using the fact that $S$ is a sphere, we get that the coboundary map $\delta$ is an isomorphism:

$$
\delta: H^{2 m+1}(S-L) \stackrel{\simeq}{\longrightarrow} H^{2 m+2}(S, S-L)
$$

Furthermore, we have the Thom isomorphism

$$
t: H^{2 m+2}(S, S-L) \stackrel{\simeq}{\longrightarrow} H^{2 m}(L)
$$

Combining the two, we get the Alexander duality isomorphism

$$
\lambda: H^{2 m+1}(S-L) \stackrel{\simeq}{\longrightarrow} H^{2 m}(L)
$$

Finally, we have a "push aside map" $\rho:=\rho_{t}: L \longrightarrow \partial X_{t} \subset S \backslash L$ that induces a map $\rho^{*}: H^{2 m+1}(S-L) \longrightarrow H^{2 m+1}(L)$. Combined we obtain a map

$$
\gamma:=\rho^{*} \circ \lambda^{-1}: H^{2 m}(L) \longrightarrow H^{2 m+1}(L) .
$$

Let finally

$$
<-,->: H^{2 m+1}(L) \times H^{2 m}(L) \longrightarrow \mathbb{Z}
$$

be the Poincaré pairing of the oriented $4 m+1$-manifold $L$. We then have the following formula.

Proposition 5.1.

$$
l k(\alpha, \beta)=<\gamma(\alpha), \beta>
$$


We mimic the above construction in topological K-theory. The pair $(S, S-L)$ defines a long exact sequence in $K$-theory as it does in cohomology. Furthermore, we have a Thom-isomorphism in $K$-theory, $T: K^{0}(S, S-L) \longrightarrow K^{0}(L)$, and the boundary map $\Delta: K^{1}(S-L) \rightarrow K^{0}(S, S-L)$ is an isomorphism as $S$ is an odddimensional sphere. Comparing with (rational) cohomology, we obtain a diagram with commuting squares:

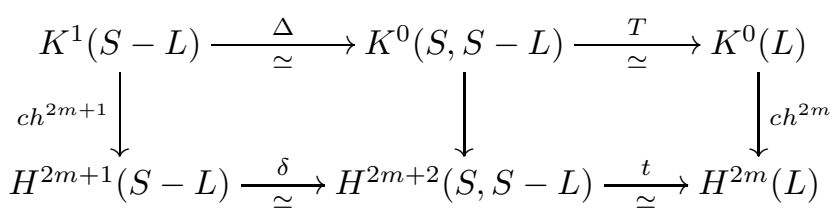

Proposition 5.2. For a maximal Cohen-Macaulay module $M$ with classes

$$
[M]_{\text {top }} \in K^{0}(L), \quad\{M\}_{\text {top }} \in K^{1}(L),
$$

one has

$$
\operatorname{ch}^{2 m+1}\left(\{M\}_{t o p}\right)=\gamma\left(\operatorname{ch}^{2 m}\left([M]_{t o p}\right)\right) .
$$

Proof: The Thom isomorphism in $K$-theory maps $E \stackrel{\alpha}{\longrightarrow} F$ on $S$, which is an isomorphism over $S-L$, to the index-bundle $[\operatorname{Coker}(\alpha)]-[\operatorname{Ker}(\alpha)]$. So if we start with the $\mathcal{O}_{X}$-resolution $0 \longrightarrow \mathcal{O}_{X}^{p} \longrightarrow \mathcal{O}_{X}^{p} \longrightarrow M \longrightarrow 0$ of $M$, then $[M]_{\text {top }} \in$ $K^{0}(L)$ is just the image of $\alpha(M) \in K^{1}(S-L)$ under $T \circ \Delta$. The geometric map $\rho_{t}: L \longrightarrow \partial X_{t}$ induces a commutative diagram

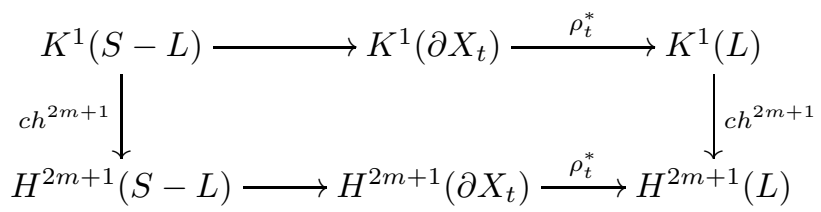

By Theorem 4.2, the element $\alpha(M) \in K^{1}(S-L)$ gets mapped to $\{M\}_{\text {top }} \in K^{1}(L)$. The formula now follows from the definition of $\gamma=\rho_{t}^{*} \circ \delta^{-1} \circ t^{-1}$.

Corollary 5.3. (Proof of the main result)

$$
\begin{aligned}
& \theta(M, N)=\quad \chi(\{M\} \otimes[N]) \quad \text { by Theorem 3.4, } \\
& =\quad \chi_{t o p}\left(\{M\}_{t o p} \otimes[N]_{t o p}\right) \quad \text { by Proposition 4.1, } \\
& =<\operatorname{ch}\left(\{M\}_{\text {top }}\right), \operatorname{ch}\left([N]_{\text {top }}\right)> \\
& =\left\langle\gamma\left(\operatorname{ch}\left([M]_{t o p}\right)\right), \operatorname{ch}\left([N]_{t o p}\right)\right\rangle \text { by Proposition 5.2, } \\
& =\quad l k\left(\operatorname{ch}\left([M]_{t o p}\right), \operatorname{ch}\left([N]_{t o p}\right)\right) \quad \text { by Proposition } 5.1 \text {. }
\end{aligned}
$$

Remark 5.4. Given a matrix factorisation $(A, B)$ for a maximal Cohen-Macaulay module $M$ one can find de Rham representatives for the Chern-classes that lie in the unstable cyclic homology of the hypersurface.

Consider $P=\mathbb{C}\left\{x_{0}, x_{1}, \ldots, x_{n}\right\}$ as a module over $\mathbb{C}\{t\}$ with $t$ acting as multiplication by $f \in P$. Denote by $\Omega^{p}$ the module of germs of $p$-forms on $\mathbb{C}^{n+1}$ and let $\Omega_{f}^{p}:=\Omega^{p} /\left(d f \wedge \Omega^{p-1}\right)$ be the module of relative differentials. One puts

$$
\omega(M):=d A \wedge d B
$$


The components of the chern-character

$$
c h_{M}:=\operatorname{tr}(\exp (\omega(M)))=\sum_{i} \frac{1}{i !} \omega^{i}(M)
$$

are well-defined classes

$$
\omega^{i}(M):=\operatorname{tr}\left((d A \wedge d B)^{i}\right) \in \Omega^{2 i} /\left(d f \wedge d \Omega^{2 i-1}\right) .
$$

There are, however, also odd-degree classes

$$
\eta^{i}(M):=\operatorname{tr}\left(A d B(d A \wedge d B)^{i}\right) \in \Omega_{f}^{2 i+1} / d \Omega_{f}^{2 i} .
$$

The group $\Omega_{f}^{2 i+1} / d \Omega_{f}^{2 i}$ can be identified with the cyclic homology $H C_{i}(P / \mathbb{C}\{t\})$. Note that there is an exact sequence

$$
0 \longrightarrow \Omega_{f}^{2 i-1} / d \Omega_{f}^{2 i-2} \stackrel{d}{\longrightarrow} \Omega^{2 i} /\left(d f \wedge d \Omega^{2 i-1}\right) \longrightarrow \Omega^{2 i} / d \Omega^{2 i-1} \longrightarrow 0
$$

and $d \eta^{i-1}(M)=\omega^{i}(M)$ by definition. If the number of variables $n+1$ is even, then a top degree form sits in the Brieskorn-module

$$
\mathcal{H}_{f}^{(0)}:=\Omega^{n+1} / d f \wedge d \Omega^{n-1},
$$

a free $\mathbb{C}\{t\}$-module of rank $=\mu(f)$, see $\underline{\mathrm{Lo}}$.

(Note however, that only in the quasi-homogeneous case its stalk at 0 ,

$$
\mathcal{H}_{f}^{(0)}(0)=\Omega^{n+1} /\left(d f \wedge d \Omega^{n-1}+f \Omega^{n+1}\right),
$$

can be identified with the jacobian ring $P / J_{f} \cong \Omega^{n+1} / d f \wedge \Omega^{n}$.)

The higher residue pairing

$$
K: \mathcal{H}_{f}^{(0)} \times \mathcal{H}_{f}^{(0)} \longrightarrow \mathbb{C}\left\{\left\{\partial_{t}^{-1}\right\}\right\}
$$

of K. Saito can be seen as the de Rham-realisation of the Seifert form of the singularity. Expressing $l k(\operatorname{ch}(M), \operatorname{ch}(N))$ in this way leads to a Kapustin-Li type formula for $\theta(M, N)$. Details of this will appear elswhere.

\section{BeHAVIOUR UNDER DEFORMATION}

As we have identified the Theta pairing $\theta(M, N)$ as a topological invariant, one would expect that this number remains constant, if we let the hypersurface and modules depend on an additional parameter $t$. We will now indicate in what sense this is indeed the case. As the arguments are well-known in singularity theory, we will be brief. We consider a one-parameter deformation of an isolated hypersurface singularity $f$ :

$$
F \in P:=\mathbb{C}\left\{x_{0}, x_{1}, \ldots, x_{n}, t\right\}, \quad F(x, 0)=f(x) .
$$

The hypersurface ring $R:=P /(F)$ is a flat module over the discrete valuation ring $\mathbb{C}\{t\}$, so we have an exact sequence

$$
0 \longrightarrow R \stackrel{t .}{\longrightarrow} R \longrightarrow \bar{R} \longrightarrow 0,
$$

where $\bar{R}:=\mathbb{C}\left\{x_{0}, \ldots, x_{n}\right\} /(f)$. If $M$ and $N$ are $R$-modules that are flat as $\mathbb{C}\{t\}$ modules, they appear similarly in exact sequences

$$
0 \longrightarrow M \stackrel{t .}{\longrightarrow} M \longrightarrow \bar{M} \longrightarrow 0,0 \longrightarrow N \stackrel{t \cdot}{\longrightarrow} N \longrightarrow \bar{N} \longrightarrow 0 .
$$


From basic principles of homological algebra it follows that there is a long exact sequence that in large enough (homological) degrees looks like

$$
\begin{aligned}
& \longrightarrow \operatorname{Tor}_{e v}^{R}(M, N) \stackrel{t .}{\longrightarrow} \operatorname{Tor}_{e v}^{R}(M, N) \longrightarrow \operatorname{Tor}_{e v}^{\bar{R}}(\bar{M}, \bar{N}) \longrightarrow \\
& \operatorname{Tor}_{\text {odd }}^{R}(M, N) \stackrel{t .}{\longrightarrow} \operatorname{Tor}_{\text {odd }}^{R}(M, N) \longrightarrow \operatorname{Tor}_{\text {odd }}^{\bar{R}}(\bar{M}, \bar{N}) \longrightarrow \cdots
\end{aligned}
$$

If length $\left(\operatorname{Tor}_{e v / o d d}^{\bar{R}}(\bar{M}, \bar{N})\right)<\infty$, then the $R$-modules $\operatorname{Tor}_{e v / o d d}^{R}(M, N)$ are finitely generated as $\mathbb{C}\{t\}$-modules. Any finitely generated $\mathbb{C}\{t\}$-module $H$ is of the form $T \oplus F$, where $T$ is the torsion submodule and $F$ is $\mathbb{C}\{t\}$-free of finite rank, $\operatorname{Rank}(H)=$ $\operatorname{Rank}(F)$. In particular,

$$
\text { length }(\operatorname{Coker}(t \cdot: H \longrightarrow H))-\operatorname{length}(\operatorname{Ker}(t \cdot: H \longrightarrow H))=\operatorname{Rank}(H) \text {. }
$$

From this we immediately obtain

\section{Corollary 6.1.}

$$
\theta(\bar{M}, \bar{N})=\operatorname{Rank}\left(\operatorname{Tor}_{\text {even }}^{R}(M, N)\right)-\operatorname{Rank}\left(\operatorname{Tor}_{\text {odd }}^{R}(M, N)\right) .
$$

Now choose an appropriate representative $p: Y \longrightarrow S$ for the hypersurface $F=0$, where $S$ is a small disc in $\mathbb{C}$. We obtain coherent sheaves $\operatorname{Tor}_{k}^{\mathcal{O}_{Y}}(M, N)$ on $Y$. Under the same assumptions these sheaves are, via $p_{*}$, coherent as $\mathcal{O}_{S}$-modules. The above $\operatorname{Rank}\left(\operatorname{Tor}_{\text {even } / \text { odd }}^{R}(M, N)\right)$ can then be identified with a sum of contributions in a general fibre over $t \in \Delta$. Hence we obtain

\section{Theorem 6.2.}

$$
\theta(\bar{M}, \bar{N})=\sum_{y \in Y_{t}} \theta_{y}\left(M_{y}, N_{y}\right),
$$

where $\theta_{y}\left(M_{y}, N_{y}\right)$ denotes Hochster's Theta pairing over the local ring of $\left(Y_{t}, y\right)$ of the localizations $M_{y}$ and $N_{y}$ at $y \in Y_{t}$, and only finitely many summands are nonzero.

Let us say that $M$ is a smoothing of $\bar{M}$ if it is locally free outside the fibre $t=0$, and call $\bar{M}$ smoothable, if it has a smoothing.

From the above theorem we then deduce:

Corollary 6.3. If $\bar{M}$ is smoothable, then $\theta(\bar{M}, \bar{N})=0$ for each $\bar{R}$-module $\bar{N}$ that extends to a $R$-module $N$ that is flat as $\mathbb{C}\{t\}$-module. In particular, $\theta(\bar{M}, \bar{M})=0 . \diamond$

To conclude, we revisit the examples 1.1. With $M=\mathcal{O}_{L}=\mathbb{C}\{x, y\} /(x)$ the maximal Cohen-Macaulay module associated to a component of the one-dimensional $A_{1}$ singularity $f=x y$, it is clear that this cycle cannot be extended to the family of hyperbolas $F=x y-t$, and the calculation $\theta(M, M)=1$ bears this out algebraically.

On the other hand, the line $L$ given by $x=z=0$ on the two-dimensional $A_{1}$ singularity $f=x y-z^{2}$ lifts to a line in a ruling on the hyperboloid $F=x y-z^{2}-t$, explaining geometrically why the associated maximal Cohen-Macaulay module $M$ satisfies $\theta(M, M)=0$, as we saw. 


\section{REFERENCES}

[AGV] V. Arnol'd, A. Varchenko and S. Guzein-Zade, Singularities of Differentiable Mappings, Vol.II, Monographs in Mathematics, Vol 83, Birkhäuser, 1988.

[A] M.F. Atiyah, K-theory. Notes by D. W. Anderson. Second edition. Advanced Book Classics. Addison-Wesley Publishing Company, Advanced Book Program, Redwood City, CA, 1989. $\mathrm{xx}+216$ pp. ISBN: 0-201-09394-4

[AB] M. Auslander and R.-O. Buchweitz, The homological theory of maximal Cohen-Macaulay approximations. Colloque en l'honneur de Pierre Samuel (Orsay, 1987). Mém. Soc. Math. France (N.S.) No. 38 (1989), 5-37.

[AvB] L. L. Avramov and R.-O. Buchweitz, Lower bounds for Betti numbers. Compositio Math. 86 (1993), no. 2, 147-158.

[B] R.-O. Buchweitz, Maximal Cohen-Macaulay Modules and Tate Cohomology over Gorenstein Rings. 155pp (1987). Available at https://tspace.library.utoronto.ca/handle/1807/16682

[D] H. Dao, Decent intersection and Tor-rigidity for modules over local hypersurfaces, arXiv:math/0611568, 2008.

[DM] T. Dyckerhoff and D. Murfet, The Kapustin-Li formula revisited, arXiv:1004.0687, 2010.

[E] D. Eisenbud, Homological algebra on a complete intersection, with an application to group representations. Trans. Amer. Math. Soc. 260 (1980), no. 1, 35-64.

[GG] H. Gillet and D. Grayson, On the loop space of the Q-construction, Illinois J. Math 31 (1987), 574-597.

[H] M. Hochster, The Dimension of an Intersection in an ambient Hypersurface. In: Algebraic geometry, Proc. Conf., Chicago Circle 1980, Lect. Notes Math. 862 (1981), 93-106.

[KL] A. Kapustin and Y. Li, Open String BRST Cohomology for Generalized Complex Branes. Adv. Theor. Math. Phys. 9 (2005), 559-574.

[Le] J. Levine, Polynomial invariants of knots of codimension two. Ann. of Math. 84 (1966), $537-554$.

[Lo] E. J. N. Looijenga, Isolated singular points on complete intersections. London Mathematical Society Lecture Note Series 77. Cambridge University Press, Cambridge, 1984. xi+200 pp. ISBN: 0-521-28674-3

[Mi] J. Milnor, Singular Points of Complex Hypersurfaces, Annals of Math. Studies 61, Princeton University Press, Princeton, N.J.; University of Tokyo Press, Tokyo 1968 iii+122 pp.

$[\mathrm{Mu}]$ D. Murfet, Residues and duality for singularity categories of isolated Gorenstein singularities. arXiv:0912.1629, 2009.

[MPSW] W. Moore, G. Piepmeyer, S. Spiroff, and W. Walker, Hochster's Theta invariant and the Hodge-Riemann Bilinear Relations. Adv. Math. 226 (2011), no. 2, 1692-1714.

[N] A. Nenashev, Double short exact sequences and $K_{1}$ of exact categories. K-theory 14 (1998), 23-41.

[O] D. O. Orlov, Triangulated categories of singularities and D-branes in Landau-Ginzburg models. Tr. Mat. Inst. Steklova 246 (2004), Algebr. Geom. Metody, Svyazi i Prilozh., 240-262; translation in Proc. Steklov Inst. Math. 2004, no. 3 (246), 227-248.

[PV] A. Polishchuk and A. Vaintrob, Chern characters and Hirzebruch-Riemann-Roch formula for matrix factorizations. 45 pages, v2, eprint arXiv:1002.2116, 2010.

[Q] D. Quillen, Higher algebraic K-theory: I. In: Higher K-theories (ed. H. Bass), Springer LNM, 341, 85-147.

[St] J. Steenbrink, Letter to R.-O. Buchweitz, $198 \%$.

[Sh1] C. Sherman, On $K^{1}$ of an abelian Category. Journal of Algebra 163, (1994), 568-582.

[Sh2] C. Sherman, Connecting homomorphisms in localization sequences, in: Algebraic K-theory (Poznań, 1995), 175-183, Contemp. Math., 199, Amer. Math. Soc., Providence, RI, 1996.

[Y] Y. Yoshino, Cohen-Macaulay modules over Cohen-Macaulay rings. London Mathematical Society Lecture Note Series, 146. Cambridge University Press, Cambridge, 1990. viii+177 pp. ISBN: 0-521-35694-6 
Dept. of Computer and Mathematical Sciences, University of Toronto at Scarborough, 1265 Military Trail, Toronto, ON M1C 1A4, Canada

E-mail address: ragnar@utsc.utoronto.ca

Fachbereich 17, AG Algebraische Geometrie, Johannes Gutenberg-Universitt, D-55099 MAinz, GeRmany

E-mail address: vanstrat@uni-mainz.de 